\title{
Rise of the appendage
}

\section{Meron Gurkiewicz ${ }^{1,2}$, Dana Iron², Izhar Bar-Gad ${ }^{1,2}$ and Alon Korngreen ${ }^{1,2 *}$}

1 The Mina and Everard Goodman Faculty of Life Sciences, Bar-Ilan University, Ramat-Gan, Israel

2 Leslie and Susan Gonda Multidisciplinary Brain Research Center, Bar-llan University, Ramat-Gan, Israel

${ }^{*}$ Correspondence: korngra@mail.biu.ac.il

Scientific publication is adapting, like other facets of everyday life, to the Internet. The rapid rise in online articles has pitted the rigid rules of scientific publication against the extreme flexibility of the Internet. In some cases these two opposing forces act in synergy while in others strange chimeras have emerged. One of these derives from the ability to make supplementary material available online as a complement to manuscripts. This comes in many forms ranging from traditional text, tables and figures, to newer multimedia formats such as soundtracks or movies. Ten years ago hardly any journals had online supplementary material. Since then there has been a steady increase in quantity but more strikingly in the number of online supplementary figures (Figure 1). The fact that researchers can enhance their manuscripts by providing online information constitutes one of the major differences between a paper bound publication and extended electronic presentations. The addition of video clips or animation to a manuscript enables the presentation of dynamic phenomena and techniques. On the plus side, adding raw data to a manuscript allows other scientists to examine the quality of the data and perhaps apply other analysis methods to the same data set. In areas such as neuroinformatics, attaching links to computer code developed for a specific study can enhance the exchange of information within the scientific community. The highly important methods section, which is currently being suffocated due to space limitations in many journals, can also thrive and allow for better reproducibility of the research when allocated the proper space in the online version of the manuscript.

Unfortunately, this new addition to scientific publications has down sides that warrant discussion by the scientific community. First, in almost all the papers

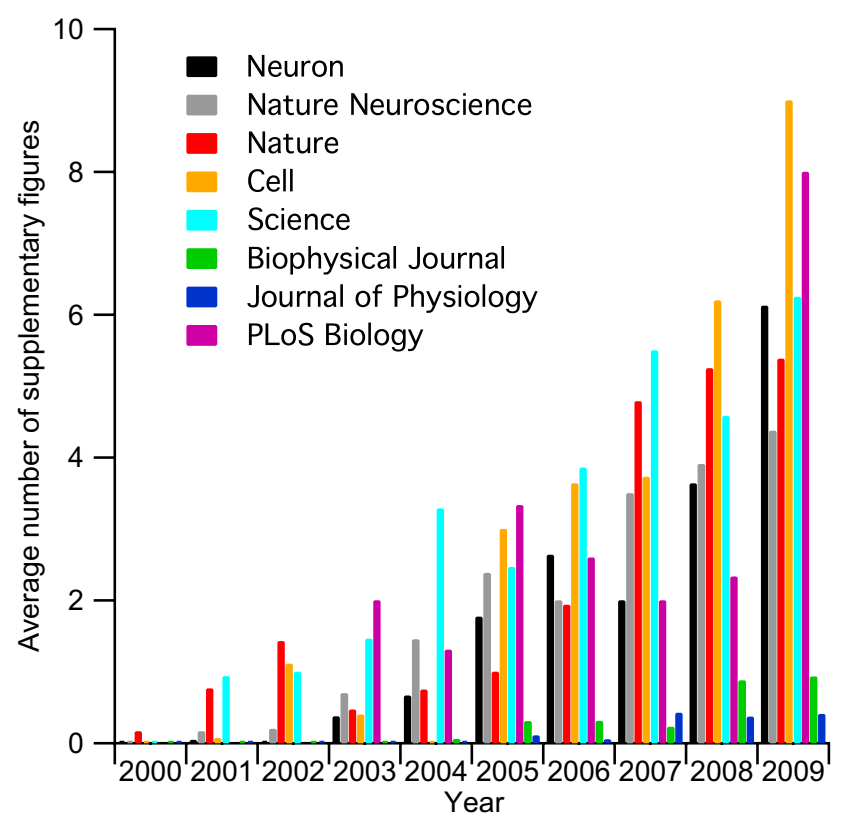

FIGURE 1 | Average number of online supplementary figures in several journals. The number of figures was calculated from the first issue of each year, not from the entire year. The journals were selected for this analysis from the pool of neuroscience and physiology journals we routinely read.

we have recently read and in those scanned in order to generate Figure 1, the formatting of the online supplementary material was at best loose and at worst sloppy. There was a considerable discrepancy between the quality of the figures appearing in the manuscript and that displayed in the online supplementary material. This evident difference in quality also applied to the supplementary text and tables that were in some cases incoherent. Oddly, the strict formatting enforced by journals to the body of the manuscript is not applied to online supplementary material. In this electronic age there should be no difference. Online supplementary material is often poorly formatted even in open-access Internet based journals that do not impose page limits on the manuscript itself. Second, the addition of online supplementary material has changed the way authors, editors and reviewers address the reviewing process. Previously, authors could always state that some of the requests made by the reviewers were beyond the scope of the manuscript and politely decline to address them. Editors respected this stance since the space in the journals was limited. The existence of online supplementary material has changed this gentleman's agreement. Today, authors prefer to address all the reviewers' requests by adding more online supplementary material. This contributes to the poor formatting and quality of online supplementary material, as many figures are made and data gathered and analyzed hastily simply to satisfy such queries. Third, since space limitations no longer exist there is a shift in the length of published manuscripts. In some journals, gargantuan papers are disguised as short communications and letters. Thus, journals with space limitations imposed by the printed edition use online supplementary material to publish larger allegedly more important manuscripts. Fourth, as a direct result of expanding online supplementary material the readability of many manuscripts has declined drastically. Manuscripts 
containing online supplementary figures are very hard to read. Jumping back and forth between the body of the paper and the supplementary section is tiring, cumbersome and truncates the flow of reading. Imagine how annoyed you might have been if Figure 1 would have been deliberately placed by us as online supplementary material. We view this as the most alarming feature of online supplementary information. Writing a scientific manuscript is like writing prose, technical and infrequently interesting but still a continuous text that should adhere to the rules of grammar, composition and fine writing. Similarly, the readability of a well written continuous text greatly contributes to the communicability of the scientific message. Not only does forcing readers to juggle several files as they read greatly reduce the reading experience but, more crucially it carries the risk of diluting the scientific message embedded in the text.

Scientific publication is attempting to escape its papery corporal body and transcend to the 'virtual' existence of the World Wide Web. The big movement toward open access publication by the Public Library of Science, the National Institute of Health, Frontiers in Neuroscience and others is one beneficial outcome of this evolutionary process. The current state of online supplementary information is less benign. Needless to say, the scientific community and publishing corporations must find a way to control the Golem born of online manuscripts so that once again we will be able to read intelligible, logical, continuous scientific prose. In the short term this can be achieved by setting limits on the size of the supplementary material section allowed per manuscript. The number of online supplementary figures and supplemental results should be limited and their quality should match material appearing in the body of the manuscript. The figures and text appearing in the supplementary sections should be better linked to the main document. Supplementary information should be typeset and formatted to achieve the same (high) level as the main manuscript. Reviewers should consider supplementary material as an integral part of the manuscript. Moreover, manuscripts published in Internet based journals have less need for a supplementary section, as all the data and text appearing there can be easily incorporated into the main text. This can be done trivially in the HTML version (even with audio and video additions) and can be incorporated as downloadable links in the PDF version. Along these same lines, journals with printed editions should have the short hard-copy version as well an online enhanced PDF.

Looking further into the future, the curious case of online supplementary information raises several important questions. Should we fully embrace electronic publication and completely eliminate the printed editions of the journals? Should the classic format of the scientific paper be preserved or should we replace it with a more sophisticated electronic version? Most importantly, what is the format for a manuscript that will enable the best transfer of the data, ideas, hypotheses and above all the state of mind of the scientist to the community? We do not yet have answers to these questions. However, we strongly feel that it is high time we stop being manipulated by the rapid changes to the Internet and decide how we want to present our science to the world.

Received: 19 August 2009; published: 08 October 2009 Citation: Front. Neuroinform. (2009) 3:32. doi: 10.3389/neuro.11.032.2009

Copyright (C) 2009 Gurkiewicz, Iron, Bar-Gad and Korngreen. This is an open-access article subject to an exclusive license agreement between the authors and the Frontiers Research Foundation, which permits unrestricted use, distribution, and reproduction in any medium, provided the original authors and source are credited. 